\section{EDUCAÇ̄̃̃O \\ V.8 $\cdot$ N.1 • Agosto/Setembro/Outubro - 2019 \\ ISSN Digital: 2316-3828 \\ ISSN Impresso: 2316-333X}

DOI: 10.17564/2316-3828.2019v8n1p23-34

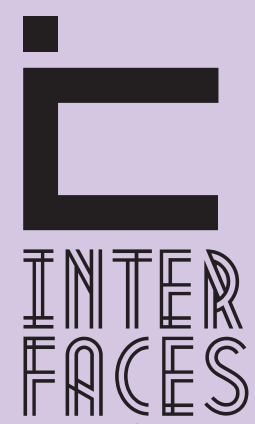

CIENTÍFICAS

\title{
ESTUDO EXPLORATÓRIO DAS TAXAS DE EVASÃO DISCENTE DO CST EM AUTOMAÇ̃̃O INDUSTRIAL DO IFS/ CAMPUS LAGARTO
}

ESTUDIO EXPLORATORIO DE LAS TASAS DE EVASIÓN DISCIDENTE DEL CST EN AUTOMACIÓN INDUSTRIAL DEL IFS/ CAMPUS LAGARTO

EXPLORATORY STUDY OF THE EVALUATION FEES OF THE CST IN INDUSTRIAL AUTOMATION OF IFS/CAMPUS LAGARTO

José Osman dos Santos ${ }^{1}$ José Espínola da Silva Junior ${ }^{2}$ Telma Amélia Santos Pereira ${ }^{3}$ Danyelle Cruz Schetine ${ }^{4}$ Igor Adriano de Oliveira Reis ${ }^{5}$

\section{RESUMO}

Atualmente, é crescente a demanda por profissionais qualificados, sobretudo para ocupação de postos ligados às Engenharias. No Brasil, a oferta de tais profissionais constitui um gargalo que tem dificultado o crescimento econômico brasileiro. Assim, o governo brasileiro tem desenvolvido ações para expansão da educação tecnológica. Entretanto, vários estudos têm evidenciado que os índices de evasão no ensino superior são significativos e de causa multifatorial. Isto posto, é importante que sejam investigados os índices de evasão e a identificação dos fatores causadores da evasão no âmbito das diversas instituições e cursos. Dessa forma, esse artigo apresenta os números relativos às taxas de evasão discente a partir do Curso Superior de Tecnologia (CST) em Automação Industrial do IFS/ Campus Lagarto e identifica algumas motivações. Dessa forma, este trabalho contribui com indicadores para orientar políticas internas para redução das taxas.

\section{PALAVRAS-CHAVE}

CST Automação Industrial. Evasão Escolar. IFS/ Campus Lagarto. 


\section{ABSTRACT}

Currently, the demand for skilled professionals is increasing, particularly for occupying positions correlated to Engineering. In Brazil, the supply of such professionals has been constituted a bottleneck for the economic growth. Thus, the Brazilian government has taken action to expand technology education. However, several studies have been shown that the rates of dropout in higher education are significant and of multifactorial origin. Consequently Therefore, it is important to investigate the rates of evasion and identification of the major factors of evasion under different institutions and courses. This way, this article presents the numbers relate to evasion rates from the Course of Technology in Industrial Automation IFS / Campus and identifies some motivations. Thus, this work contributes with indicators to guide internal policies to reduce the evasions rates and reducing the social cost of this phenomenon.

\section{KEYWORDS}

CST Industrial Automation. School Dropout. IFS/Campus Lagarto.

\section{RESUMEN}

Actualmente, es creciente la demanda por profesionales calificados, sobre todo para la ocupación de puestos vinculados a las Ingenierías. En Brasil, la oferta de tales profesionales constituye un cuello de botella que ha dificultado el crecimiento económico brasileño. Así, el gobierno brasileño ha desarrollado acciones para la expansión de la educación tecnológica. Sin embargo, varios estudios han evidenciado que los índices de evasión en la enseñanza superior son significativos y de causa multifactorial. Esto puesto, es importante que se investiguen los índices de evasión y la identificación de los factores causantes de la evasión en el ámbito de las diversas instituciones y cursos. De esta forma, este artículo presenta los números relativos a las tasas de evasión discente a partir del Curso Superior de Tecnología (CST) en Automatización Industrial del IFS/Campus Lagarto e identifica algunas motivaciones. De esta forma, este trabajo contribuye con indicadores para orientar políticas internas para reducción de las tasas.

\section{PALABRAS CLAVES}

CST Automatización Industrial. Evasión Escolar. IFS / Campus Lagarto. 


\section{INTRODUÇÃO}

Em virtude do processo de estabilização da economia no Brasil após os anos 1990, que implicou em uma forte taxa de crescimento econômico, o país passou a conviver as ameaças do "apagão" na oferta de mão de obra e, consequentemente, da sustentabilidade do ritmo de crescimento do PIB, em particular de mão de obra especializada, própria de um mundo globalizado, altamente competitivo e pautado no conhecimento (MENEZES-FILHO; RODRIGUES JR., 2003). Esse novo panorama econômico e político, que implicou em uma competitividade entre as empresas e uma forte pressão por produtividade e qualidade nos produtos e serviços, tem um reflexo na procura por mão de obra qualificada, principalmente em setores específicos, como: os setores de petróleo e gás, telecomunicações e indústria de transformação.

Atribuem-se, tendo em vista esse processo de crescimento econômico e expansão das fronteiras comerciais, que está associada à procura por profissionais de nível superior, novas metas, responsabilidade e desafios aos Estados, aos indivíduos e à sociedade, especialmente, como consequência da flexibilização nos processos de produção e da gestão do trabalho, que requer um perfil profissional diferenciado (GUSSO et al., 2011).

Assim, a produção tem exigido um trabalho de arbitragem, onde os indivíduos devem ser capazes de diagnosticar, antecipar, analisar, sintetizar, trabalhar em grupo, tomar decisões e participar ativamente do processo, exercendo sua autonomia intelectual. Sob esta ótica e considerando a necessidade de implantação de sistemas de produção que otimizem a competitividade, os parques industriais têm adotado dinâmicas e formas de gestão que objetivam a substituição do trabalho humano pela eficiência e perfeição da alta tecnologia, com vistas à automatização dos processos.

Essas características de mercado e do sistema produtivo impõem uma formação inicial e continuada de profissionais de alta qualificação. Constituindo assim, em um desafio aos mercados emergentes, como o brasileiro, os quais são carentes de uma mão de obra com um perfil desejado aos novos paradigmas industriais que estão se estabelecendo. Nesse panorama, tem sido apontado que a ampliação e consolidação do Brasil no comércio internacional têm sido estranguladas pela ausência de qualificação do trabalhador, principalmente, em relação a uma sólida formação alicerçada nas inovações tecnológicas, lideradas pelas áreas da informática e da microeletrônica (DZIEKANIAK; ROVER, 2011; ROCHA-VIDIGAL; VIDIGAL, 2012; GOMES, 2013).

Este gargalo que impede o crescimento econômico brasileiro, em uma taxa além dos atuais $4 \%$, já era visto com grande preocupação nos anos 1990, onde uma Pesquisa Nacional por Amostra de Domicílios - 1990 (PNAD/90), realizada pelo Instituto Brasileiro de Geografia e Estatística (IBGE), indicavam que $53 \%$ da força de trabalho (cerca de 33 milhões de trabalhadores), tinham até cinco anos de permanência na escola, contrariando análises internacionais que recomendavam que fossem necessários no mínimo oito anos para treinamentos específicos (IBGE, 1992). Destarte, o país tem um enorme obstáculo a ser superado: qualificar trabalhadores, assegurando-lhes empregos de qualidade e garantir o processo de modernização da produção, que tem forte presença da automação nos segmentos da instrumentação e controle dos processos industriais (MACIENTE; ARAUJO, 2011). 
Frente a essas necessidades e com base na Lei 9.394/96-LDB, os cursos superiores de tecnologia têm buscado atender as demandas da sociedade brasileira quanto à formação qualificada do trabalhador, com maior flexibilidade e adaptabilidade às flutuações do mercado de trabalho (BRASIL, 1996). Nesse sentido, o Curso Superior de Tecnologia em Automação Industrial, foi implantado na Unidade de Ensino Descentralizada de Ensino de Lagarto, hoje IFS/SE - campus Lagarto, a partir do segundo semestre do ano 2007, oferecendo uma alternativa para a formação de novos profissionais na área da automação industrial, preparando profissionais para o entendimento, utilização e adaptação de novas tecnologias.

A formação de tecnólogos em automação industrial no Campus Lagarto tem contribuído para redução da razão demanda/oferta de profissionais na área e está alicerçada na concepção de profissionais com competências necessárias para empreender negócios próprios ou transferir as competências adquiridas, empreendendo projetos em indústrias nos setores químicos, alimentícios, petroquímicos, eletromecânicos, produção de mecânica, distribuição e controle de energia elétrica, entre outros ramos.

Segundo dados da Federação das Indústrias do Estado de Sergipe (FIES), o desempenho econômico do Estado de Sergipe apresentou crescimento superior às médias Nacional e Regional, no período compreendido entre 1995 e 2005, uma vez que a taxa de crescimento da economia sergipana foi de 3,3 \% a.a contra 2,7 \% a.a do Brasil e 3,0 \% a.a do Nordeste. Este comportamento da economia sergipana tem sido influenciado, principalmente, pelo desenvolvimento industrial, cuja participação média anual no Produto Interno Bruto (PIB), no período 1995 - 2005 foram de 53,2\%, com taxa média anual de crescimento de 4,2\%. De acordo com estes números, é apontado que o Estado de Sergipe apresenta características que o distingue dos demais estados do Nordeste e das demais regiões do Brasil, pois no ano de 2004, por exemplo, 53,9\% da riqueza produzida em Sergipe estiveram correlacionadas ao setor industrial (FIES, 2007).

Dentro da perspectiva da crescente expansão industrial, no Estado de Sergipe, torna-se necessária a formação de profissionais especializados, uma vez que os processos do setor se apresentam, predominantemente automatizados. Dessa forma, a área de Automação Industrial se destaca como a mais carente de profissionais do referido setor. Diante disso, o IFS-SE concatenado com as necessidades da indústria sergipana e sendo uma Instituição comprometida com o ideal da inclusão social, por meio da implantação da educação tecnológica, compromete-se em proporcionar à sociedade uma graduação tecnológica em Automação Industrial.

As grandes unidades industriais do Estado de Sergipe estão ligadas aos arranjos e cadeias produtivas dos segmentos de: petróleo e gás, fertilizantes, cerâmicas de revestimentos, têxteis e confecções, alimentos e bebidas e da construção civil. Neste sentido foi justificada a abertura do curso de Tecnologia em Automação Industrial, visando atender a uma clientela de egressos do ensino médio e técnicos de nível médio nas áreas de: Eletromecânica, Eletrotécnica, Eletrônica e Informática, que visam atuar: na análise de projetos, operação e manutenção, relacionadas a área de automação industrial.

O Instituto Federal de Sergipe é a instituição responsável pela expansão da educação tecnológica dentro das fronteiras do nosso Estado. Devido a sua tradição no setor e por reunir as melhores 
condições para tal, ressaltando-se as físicas como a qualificação dos docentes, podemos destacar os espaços criados para o desenvolvimento de tecnologias voltadas para este fim: o laboratório de automação industrial do campus Lagarto e os laboratórios de informática, os quais são utilizados como simuladores de sistemas autômatos.

Outra característica da instituição, fator relevante, é a característica do seu corpo docente, que conta com diversos doutores, mestres e especialistas na área abordada. Diante dessa situação anteriormente elencada e com os requisitos necessários para a implantação do CST Automação Industrial, no segundo semestre do ano de 2007, o Campus Lagarto do IFS foi contemplado com a aprovação pelo Conselho Superior do IFS com o curso em tela.

Apesar da alta demanda por profissionais para atuação nas diversas áreas que exigem a automatização de processos, além da relativa alta na procura pelo curso e a avaliação bem sucedida pelo Instituto Nacional de Estudos e Pesquisas Educacionais Anísio Teixeira (INEP) (nota 4 em 2010), o curso vem apresentando números desfavoráveis, relativos ao índice de evasão. Ainda que a evasão nos cursos superiores, não seja um problema exclusivamente nacional, uma vez que os índices de evasão têm uma forte contribuição nos resultados alcançados nos sistemas educacionais, a evasão preocupa o poder público, pois implica em desperdícios sociais, acadêmicos e econômicos.

O fenômeno da evasão nas Instituições de Ensino Superior no Brasil é ainda mais preocupante nos cursos relacionados à Engenharia, Ciências, Matemática e Computação, flutuando em torno de 25 \%, superior à média nacional para outras áreas, quando levado em conta estudos baseados em dados do INEP no período de 2001-2005 (SESu/MEC 1996; SILVA et al., 2007; SANTOS et al., 2011). Dessa forma, fica evidente que a evasão é mais acentuada nos cursos de tecnologia e, principalmente, nos dois primeiros semestres, em virtude da alta retenção das disciplinas de formação básica.

Nos cursos superiores de tecnologia, em especial no Curso Superior de Tecnologia em Automação Industrial, há registros que os índices de evasão e retenção têm sido bastante elevados: flutuando em torno de $60 \%$, como os casos dos cursos do IFSC e CEFET-RJ, o qual, a princípio, não difere acentuadamente dos números registrados no Instituto Federal de Sergipe (GUIMARÃES, 2012; REIS et al., 2012).

Diversos estudos têm evidenciado que os índices de evasão no ensino superior são de causa multifatorial, sendo que os fatores socioeconômicos são de maior influência na explicação da evasão (Martins, 2007; Rodriguez, 2011). Assim, é importante que sejam investigados os índices de evasão e a identificação dos fatores causadores da evasão no âmbito das diversas instituições e cursos. Por mais que se pesquisem os fatores determinantes da evasão discente, percebe-se que não há um padrão ou lógica uniforme que explique as razões da evasão. Constata-se que há que poucas pesquisas que objetivam elucidar as razões que levam os discentes a "abandonar" um curso de graduação. Assim, faz-se necessário o estabelecimento de uma sistemática de avaliação que permita diagnosticar esta situação em diferentes regiões do país e cursos.

Assim, este trabalho visa analisar os números relativos à evasão e a situação acadêmica dos alunos do Curso Superior de Tecnologia em Automação Industrial do IFS, de forma a entender o fenômeno da evasão e retenção de alunos do curso. Foram identificados alguns fatores responsáveis 
pela evasão, de forma a orientar o planejamento das ações afirmativas para consolidação do curso, buscando apontar caminhos para reduzir a evasão/retenção.

Acreditamos que pensando estas questões, isto resultará em ganhos na formação dos egressos deste curso e, principalmente, em relação às expectativas dos setores produtivos que integram as tecnologias mecânicas, microinformáticas e eletroeletrônicas em seus processos. Uma vez que ampliarão os horizontes de aplicações destas tecnologias nos diversos tipos de indústrias e, consequentemente, contribuindo para consolidação do desenvolvimento econômico e social do Estado de Sergipe e demais regiões.

\section{MATERIAL E MÉTODOS}

Com objetivo de obter os dados de evasão/retenção e verificar alguns fatores que têm influência quanto ao processo de fracasso escolar e desistência dos Cursos Superior de Tecnologia em Automação Industrial do IFS/Campus Lagarto, inicialmente foi definido, em conjunto com CRE/Campus Lagarto (Coordenadoria de Registro Escolar) a amostra de estudantes e egressos a serem entrevistados e encaminhamentos em relação ao acesso a base de dados daquele setor, bem como do setor de Serviço Social do Campus. Fizeram parte da amostra estudantes que ingressaram na instituição no período de 2007/2 a 2011/1, totalizando uma amostra de 340 discentes. Quanto às escolhas metodológicas, o projeto é de natureza quantitativa, descritiva-correlacional e exploratória, e foi desenvolvido nas distintas etapas a seguir:

1. Obtenção dos números absolutos relativos aos alunos egressos, matriculados, trancados, retenções (disciplinas e períodos) e transferências. Esses dados foram consultados a partir do Sistema Acadêmico, em colaboração com a Coordenadoria de Registro Escolar, referente ao período 2007-2011;

2. Elaboração e distribuição de questionários, similar àqueles aplicados pelo INEP diante da realização do ENADE, de forma a estabelecer o perfil socioeconômico do discente do Curso de Automação Industrial. Após aplicação do questionário, 57 discentes responderam e devolveram o questionário a respeito da sua satisfação quanto ao curso e dados socioeconômicos, correspondendo a $17 \%$ do total de estudantes. Predominantemente, os alunos que devolveram os questionários foram os alunos ainda matriculados, pois os alunos evadidos ainda não devolveram o questionário;

3. Elaboração e distribuição eletrônica ou envio para endereços, ou agendamento de entrevistas, para os alunos evadidos, o qual contém questões que busquem identificar os motivos que os levaram a evadir-se do curso, assim como traçar o perfil socioeconômico do evadido;

4. Exploração da base de dados por meio da aplicação de técnicas estatísticas univariadas (medidas de tendência central das variáveis, dispersão, regressões, correlações, entre outras) e multivariadas (regressão múltipla, análise fatorial etc.);

5. Caracterização dos perfis dos discentes e evadidos;

6. Identificação dos principais fatores que contribuem com a evasão e retenção dos alunos. 


\section{RESULTADOS E DISCUSSÃO}

A partir dos dados coletados, foi verificado que o corpo discente do Curso Superior de Tecnologia em Automação Industrial do IFS/Campus Lagarto é constituído predominantemente por pessoas do sexo masculino (82\%), resultado similar ao encontrado por Vasconcelos \& Brisola (2009), os quais apontam que na Universidade de Campinas (UNICAMP) menos de $30 \%$ de estudantes são do sexo feminino, optam por cursos de Ciências Exatas e Engenharias. Esse quadro pode ser representado, também, de uma forma mais crítica para mulheres atuando na pesquisa nas Engenharias Elétrica e Mecânica, que tem forte correlação com o CST Automação Industrial, onde apenas 13\% são do sexo feminino (LOMBARDI, 2005).

Essa distribuição de discentes por sexo pode ser explicada, parcialmente, por paradigmas de fundo cultural, histórico e social, que ainda permitem preconceitos arraigados em nossa sociedade, os quais precisam ser alterados, para permitir as mesmas oportunidades, independentemente do gênero, uma vez que a divisão do trabalho por gênero tem se tornado cada vez mais dinâmico, flexível e plural (KLANOVICZ, 2010).

Por meio da análise da Figura 1, constata-se que a evasão por sexo é proporcional ao número de ingressantes por sexo, ou seja, a evasão independe do gênero. A evasão total a partir do CST Automação Industrial do IFS/Campus Lagarto é de cerca de $71 \%$ do total de alunos ingressos, sendo que, aproximadamente, $70 \%$ dos ingressantes ( $72 \%$ dos homens e $69 \%$ das mulheres) de cada sexo abandonam o curso. 0 tipo de comportamento demonstrado na Figura 1, nos leva a inferir que o fator gênero não influência na taxa de evasão do curso em tela.

Figura 1 - Evasão por gênero para amostra estudada
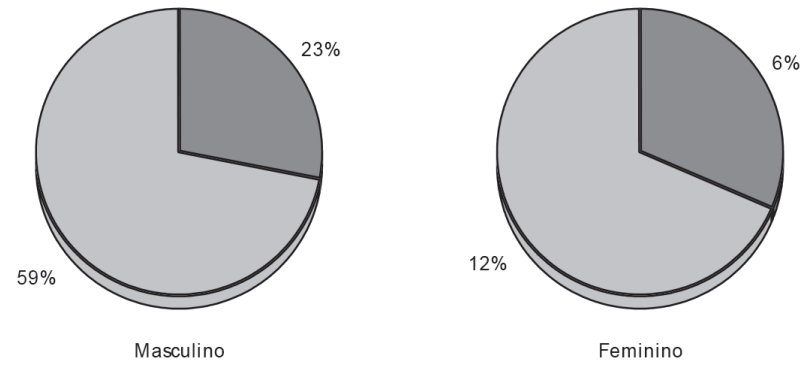

Evasão por sexo

Fonte: Dados da pesquisa.

Por meio da interpretação estatística dos dados coletados nesta pesquisa, foi possível detectar que, cerca de $76 \%$ dos alunos que abandonaram o curso (FIGURA 2), o fizeram nos dois primeiros períodos do curso, resultado similar ao encontrado na literatura (BARROSO; FALCÃO, 2004), e apenas $6 \%$ a partir da segunda metade do curso. 
De acordo com a literatura, é possível observar que o fenômeno da evasão nas Instituições de Ensino Superior no Brasil é ainda bastante preocupante nos cursos relacionados à Engenharia, Ciências, Matemática e Computação, flutuando em torno de $25 \%$, superior à média nacional para outras áreas, quando levado em conta estudos baseado em dados do INEP no período de 2001-2005 (SILVA-FILHO et al., 2007). É evidente que a evasão é mais acentuada nos cursos de tecnologia, principalmente nos dois primeiros semestres, em virtude da alta retenção das disciplinas de formação básica, sobretudo aquelas relacionados com os saberes matemáticos.

Nos cursos superiores de tecnologia, em especial no Curso Superior de Tecnologia em Automação Industrial, há registros que os índices de evasão e retenção têm sido bastante elevados, flutuando em torno de $60 \%$, como os casos dos cursos do Instituto federal de Santa Catarina (IFSC) e CEFET-RJ, o qual, a princípio, não difere acentuadamente dos números registrado no CST Automação IFS/Campus Lagarto.

Assim, na Figura 3 observa-se que a taxa de evasão no CST Automação Industrial do Campus Lagarto, que é de cerca de $70 \%$ e tem uma tendência de queda ao longo dos anos. Essa queda na taxa de evasão, apesar de pequena, pode ser explicada pela alteração na matriz curricular que ocorreu no segundo período do ano de 2008, a qual inseriu um semestre adicional ao curso, com objetivo de inserir na matriz curricular saberes básicos de matemática elementar e lógica de programação, disciplinas que apresentam uma alta taxa de retenção.

Ainda é importante citar que a evasão escolar é um fenômeno multifatorial e a evasão nos primeiros semestres têm uma forte relação com a adaptação na vida acadêmica, questões socioeconômicas, escolha do curso, organização didática da instituição, desvalorização da profissão, participação em programas institucionais e disciplinas de alto índice de retenção. Neste estudo, obteve-se que as disciplinas de cálculo I e Fundamento de Matemática apresentam taxa de retenção de mais de 75\%, seguido pela disciplina Algoritmo com retenção de cerca de 70\%. Em regra, os alunos que ficaram retidos abandonaram o curso nos semestres iniciais.

Figura 2 - Evasão/ período no CST Automação Industrial com referência ao período de abandono

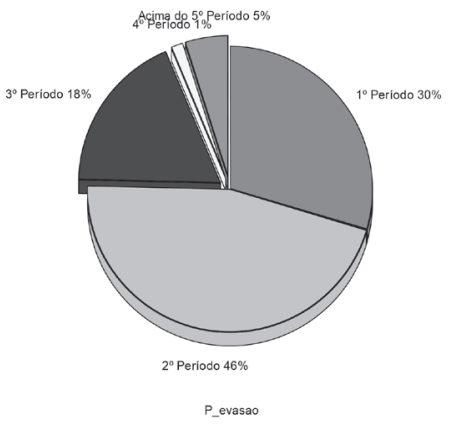

Fonte: Dados da pesquisa. 
Figura 3 - Taxa de evasão do CST em Automação Industrial em função do período de ingresso do discente

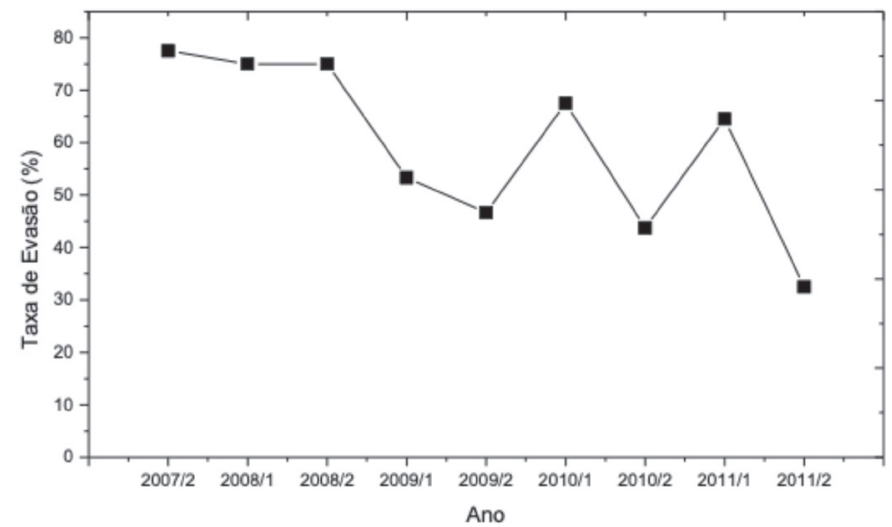

Fonte: Dados da pesquisa.

Em busca de encontrar os fatores que explicam as altas taxas de evasão no CST em Automação Industrial no IFS/Campus Lagarto, foram aplicados questionários para avaliar o nível de satisfação do discente com o curso. Na Figura 4, é possível observar que apenas 5\% dos estudantes estão plenamente satisfeitos com o curso, considerando fatores com a infraestrutura da instituição, organização didática, apoio assistencial ao aluno e comportamento dos docentes diante das dificuldades de aprendizagem dos alunos.

Quando perguntado a motivação da insatisfação, 68\% responderam que predominantemente que a motivação é de ordem institucional (relacionados aos laboratórios, biblioteca, currículo, oportunidade de estágios e participação em PIBIC, entre outras). Dessa forma, é necessário o desenvolvimento de ações estruturantes e afirmativas, para reduzir as altas taxas de evasão a partir do curso, pois os fatores apontados com mais frequência estiveram correlacionados com a visão que o discente tem da instituição e de sua capacidade financeira para continuar no curso.

Figura 4 - Nível de satisfação do discente do discente do CST em Automação Industrial

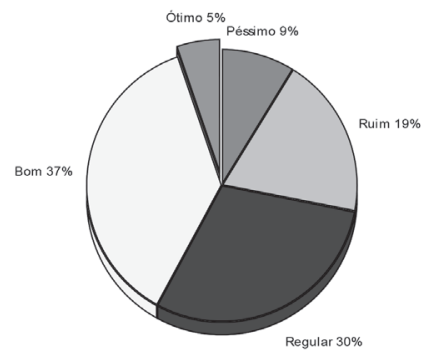

Fonte: Dados da pesquisa. 
Outro motivo de interesse que foi detectado na pesquisa esteve correlacionado com a preocupação e insegurança quanto ao não reconhecimento do curso de uma forma ampla pelos conselhos de classes e pelos setores produtivos, gerando expectativas negativas quanto à absorção no mercado de trabalho. Dessa forma, é necessária uma política governamental de apoio ao egresso dos cursos tecnológicos, bem como sua regulamentação no mercado de trabalho, pois a alta evasão implica em um elevado custo social, já que a evasão de um aluno implica na impossibilidade da entrada de outro e eleva o custo aluno.

Assim, faz-se necessária a implantação de uma política de combate a evasão no IFS, dotadas de estratégias que envolvam toda comunidade acadêmica, inclusive com programas de capacitação de gestores para que eles adquiram uma visão sistêmica para identificar causas e traçar estratégias de ações para otimizar os investimentos governamentais empregados na formação profissional.

\section{CONCLUSÕES}

O fracasso acadêmico implica em desperdício de recursos públicos, desgastes da imagem institucional e em virtude da forte demanda por profissionais da área de automação industrial no país, a exclusão da possibilidade de formação causa insegurança quanto ao número de profissionais que a sociedade tem demandado para um desenvolvimento industrial sustentável. Esse trabalho identificou que a taxa de evasão de discentes do CST Automação Industrial do Campus Lagarto é muito elevada, aproximadamente $70 \%$, independente de sexo e está correlacionada principalmente com fatores institucionais e socioeconômicos.

O trabalho contribui para ampliar a discussão das políticas institucionais que visem aumentar a taxa de formação de discentes do curso em tela, pois se faz necessário estabelecer estratégias para utilização dos gastos públicos para formação de profissionais da área de tecnologia. Pois, frente ao crescente desenvolvimento econômico e industrial no Brasil, é importante combater o fracasso escolar, visto que os países que têm maior capacidade para gerar conhecimento científico - tecnológico e educação de qualidade conseguem atrair maiores investimentos, contribuindo para a solidificação da economia, da geração de empregos qualificados e melhoria das condições de vida da população.

\section{REFERÊNCIAS}

BARROSO, M. F.; FALCÃO, E. B. M. Evasão universitária: o caso do instituto de física da UFRJ.

Encontro Nacional de Pesquisa em Ensino de Física, 9, 2004. Anais[...], Jaboticatubas, 2004.

BRASIL. Lei de Diretrizes e Bases da Educação Nacional (Lei no 9394/96). Brasília: MEC/SEMTEC, 1996.

Comissão especial de estudos sobre a evasão nas universidades públicas. Diplomação, retenção e evasão nos cursos de graduação em instituições de ensino superior públicas. Brasília: SESu/MEC, 1996. 
DZIEKANIAK, G.; ROVER, A. Sociedade do conhecimento: características, demandas e requisitos. Revista de Informação, v. 12, n. 5, out. 2011.

FIES. Sergipe: Dinâmica recente e perspectivas do setor industrial. Aracaju, 2007.

GOMES, C. A. Uma crise do capital humano na economia brasileira. Perspectivas em Gestão \& Conhecimento, João Pessoa, v. 3, n. 1, jan./jun. 2013.

GUIMARÃES, S. L. Trabalho pedagógico como possibilidade frente à evasão escolar em um curso superior de tecnologia. 2012. Dissertação (Mestrado em Educação) - Faculdade de Educação da Universidade Estadual de Campinas, Campinas, 2012.

GUSSO, D. A.; NASCIMENTO, P. A. M. M. Contexto e dimensionamento da formação de pessoal técnico científico e de engenheiros. Radar, Brasília: Ipea, n. 12, fev. 2011.

IBGE. Pesquisa Nacional por Amostra de Domicílios - 1990. Rio de Janeiro: IBGE, 1992.

KLANOVICZ, L. R. F. Gênero e Engenharias: Estudo histórico quali-quantitativo da inserção, permanência e produção científica de mulheres no Sul do Brasil. Anais de Fazendo Gênero 9 Diásporas, Diversidades e Deslocamentos, 2010.

LOMBARDI, M. R. Perseverança e resistência: a engenharia como profissão feminina. 2005. Tese (Tese de doutorado), Faculdade de Educação-Universidade Estadual de Campinas, Campinas. 2005.

MACIENTE, A. N.; ARAÚJO, T. C.. A demanda por engenheiros e profissionais afins no mercado de trabalho formal. Radar, Brasília: Ipea, n. 12, fev. 2011.

MARTINS, C. B. N. Evasão de alunos nos cursos de graduação em uma instituição de ensino superior. 2007. Dissertação (Mestrado em Administração) - Fundação Dr. Pedro Leopoldo, Pedro Leopoldo. 2007.

MENEZES - FILHO, N. A.; RODRIGUES JR., M. Tecnologia e demanda por qualificação na indústria brasileira. Revista Brasileira de Economia, Rio de Janeiro, v. 57, n. 3, jul./set. 2003.

REIS, V. W.; CUNHA, P. J. M.; SPRITZER, I. M. P. A. Evasão no ensino superior de engenharia no Brasil: um estudo de caso no CEFET/RJ. Congresso de Educação em Engenharia, 40, 2012. Anais[...], Belém, 2012.

ROCHA-VIDIGAL, C. B.; VIDIGAL, V. G. Investimento na qualificação profissional: uma abordagem econômica sobre sua importância. Acta Scientiarum. Human and Sciences, Maringá, v. 34, n. 1, jan./jun. 2012. 
RODRIGUEZ, A. Fatores de permanência e evasão de estudantes do ensino superior privado brasileiro. Simpósio de Excelência em Gestão e Tecnologia, 8, 2011. Anais[...], São Paulo, 2011.

SANTOS, B. C. A.; LOPES, D. A. Evasão e avaliação institucional no ensino superior: uma discussão bibliográfica. Avaliação, Campinas, v. 16, n. 2, jul. 2011.

SILVA FILHO, R. L. L.; MOTEJUNAS, P. R.; HIPÓLITO, O.; LOBO, M. B. C. M. A evasão no ensino superior brasileiro. Cadernos de Pesquisa, v. 37, n. 132, p. 641-659, 2007.

VASCONCELLOS, E. C. C., BRISOLLA, S. N. Presença feminina no estudo e no trabalho da ciência na UNICAMP. Cadernos Pagu, Campinas, v. 32, jan./jun. 2009.

1 Professor doutor permanente do Programa de Pós-graduação do Mestrado Profissional em Educação Profissional e Tecnológica (ProfEPT). E-maill: osmansantos@ig.com.br

2 Professor doutor permanente do Programa de Pós-graduação do Mestrado Profissional em Educação Profissional e Tecnológica (ProfEPT) E-mail: osmansantos@ig.com.br

3 Mestre em Educação pela Universidade Federal de Sergipe, Pedagoga do Instituto Federal Educação, Ciência e Tecnologia de Sergipe - IFS. E-mail: telma.pereira@ifs. edu.br

4 Professor doutor permanente do Programa de Pós-graduação do Mestrado Profissional em Educação Profissional e Tecnológica (ProfEPT) E-mail: reisigoradriano@gmail.com

5 Mestranda do Programa de Pós-graduação do Mestrado Profissional em Educação Profissional e Tecnológica (ProfEPT), Instituto Federal Educação, Ciência e Tecnologia de Sergipe - IFS. E -mail: danyelleflorzinha@hotmail.com
Recebido em: 4 de Abril de 2018

Avaliado em: 3 de Maio de 2018

Aceito em: 3 de Maio de 2018

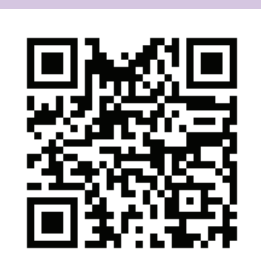

A autenticidade desse artigo pode ser conferida no site https://periodicos. set.edu.br

\section{Como citar este artigo:}

ROMEO, Andrea. Lo special account del fenomeno religioso nel dibattito nordamericano. Argumenta Journal Law, Jacarezinho - PR, Brasil, n. 29., 2018, p. 15-48. DOI: 10.17564/2316-3828.2018v7n1p13-24

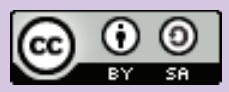

Este artigo é licenciado na modalidade acesso abertosob a Atribuição-Compartilhalgual CC BY-SA

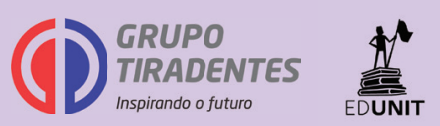

